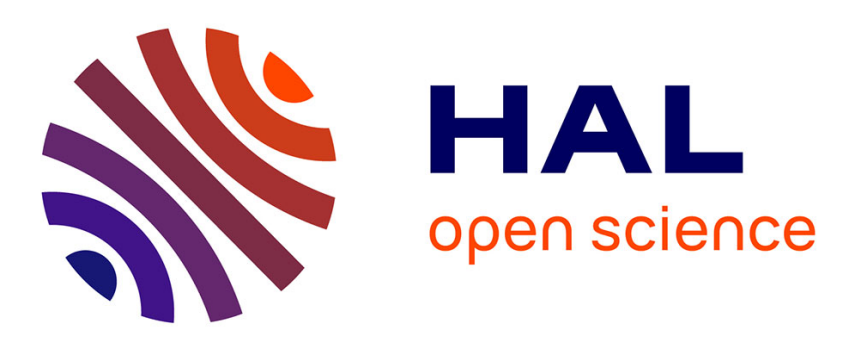

\title{
Doped Lead Halide White Phosphors for Very High Efficiency and Ultra-High Color Rendering
}

Hailong Yuan, Florian Massuyeau, Nicolas Gautier, Antoine Blaise Kama, Eric Faulques, Fei Chen, Qiang Shen, Lianmeng Zhang, Michaël Paris, Romain Gautier

\section{To cite this version:}

Hailong Yuan, Florian Massuyeau, Nicolas Gautier, Antoine Blaise Kama, Eric Faulques, et al.. Doped Lead Halide White Phosphors for Very High Efficiency and Ultra-High Color Rendering. Angewandte Chemie International Edition, 2020, 59 (7), pp.2802-2807. 10.1002/anie.201910180 • hal-02553666

\section{HAL Id: hal-02553666 https://hal.science/hal-02553666}

Submitted on 30 Apr 2020

HAL is a multi-disciplinary open access archive for the deposit and dissemination of scientific research documents, whether they are published or not. The documents may come from teaching and research institutions in France or abroad, or from public or private research centers.
L'archive ouverte pluridisciplinaire HAL, est destinée au dépôt et à la diffusion de documents scientifiques de niveau recherche, publiés ou non, émanant des établissements d'enseignement et de recherche français ou étrangers, des laboratoires publics ou privés. 


\title{
Doped Lead Halide White Phosphors for Very High Efficiency and Ultra High Color Rendering
}

\author{
Hailong Yuan ${ }^{[a]}[b]$, Florian Massuyeau ${ }^{[b]}$, Nicolas Gautier ${ }^{[b]}$, Antoine Blaise Kama ${ }^{[b]}$, Eric Faulques ${ }^{[b]}$, Fei \\ Chen ${ }^{[a]}$, Qiang Shen ${ }^{[a]}$, Lianmeng Zhang ${ }^{[a]}$, Michael Paris ${ }^{[b]}$ and Romain Gautier ${ }^{*[b]}$
}

\begin{abstract}
Lighting with ultra-high color rendering is recently drawing more attention for applications such as jewellery, photography, museum and surgery but also in daily use as the intense blue emission from the commercial white LEDs was found responsible of negative effects on the body's circadian clock. Near-UV-pumped white light-emitting diodes with ultra-high color rendering and reduced blue light emission would be a solution to this issue. However, discovering a single-phase white light emitter with such characteristics remains challenging. Herein, we demonstrate that $\mathrm{Mn}$ doping as low as $0.027 \%$ in the hybrid post-perovskite type (TDMP)PbBr4 enables to achieve a bright pure white emission replicating the spectrum of the Sun'rays. Thus, a white phosphor exhibiting an emission with CIE coordinates $(0.330,0.365)$, a high photoluminescence quantum yield of $60 \%$ (new record for white light emission of hybrid lead halides), and an ultra-high color rendering index $(C R I=96, R 9=91.8)$, corresponding to the record value for a single phase emitter was obtained. The investigation of the photoluminescence properties revealed how free excitons, selftrapped excitons and low amount of Mn dopants are coupled to give rise to such pure white emission.
\end{abstract}

\section{Introduction}

White light-emitting diodes (wLEDs), which are regarded as the next-generation lighting sources, have been commonly used in diverse applications such as daily lighting, computers and mobile phones. ${ }^{[1-3]}$ Nowadays, a blue chip (InGaN) coated with yellow phosphors (YAG: $\mathrm{Ce}^{3+}$ ) is widely used to produce the commercial wLEDs. ${ }^{[4,5]}$ However, such design has a drawback: the emission of blue light originating from the blue chip is much more intense than the one of yellow light originating from phosphors. ${ }^{[6,7]}$ Artificial blue-rich white light has been reported to have negative effects on sleep-wake cycles, eating patterns, metabolism, and mental alertness as the photo-sensitive ganglion cells in the retina signal the brain to stop producing melatonin. ${ }^{[8-10]}$ Hence, the blue light emission for LEDs lighting technology is becoming an important concern for public health.

Filtering out the excess of blue light without influencing the output white light quality is complex for wLEDS based on blue chip. Nevertheless, such filtering is possible for near UVpumped wLEDs, as UV light does not directly contribute to the white light. ${ }^{[11]}$ Hence, a near-UV LED coated with a single-phase broadband emitter to produce white light is desired. Compared with phosphors mixtures, a single-phase white emitter avoids

[a] H.L. Yuan, F. Chen, Q. Shen, L.M. Zhang

State Key Lab of Advanced Technology for Materials Synthesis and Processing, Wuhan University of Technology, Wuhan 430070 , China.

[b] H.L. Yuan, F. Massuyeau, N Gautier, A. B. Kama, E. Faulques, M. Paris and R. Gautier

Institut des Matériaux Jean Rouxel, Université de Nantes, CNRS, 2 rue de la Houssinière B.P. 32229, 44322 Nantes cedex 3, France. E-mail: Romain.Gautier@cnrs-imn.fr

Supporting information for this article is given via a link at the end of the document. any issues of reabsorption between different phosphors, the different particle sizes and nonuniformity of luminescence properties and different aging of the materials. ${ }^{[12,13]}$

Recently, low dimensional hybrid metal halides including hybrid perovskites have attracted a great attention. ${ }^{[14-17]}$ Their emissions can be extremely broad owing to exciton self-trapping as originally reported by Nagami et al. for a hybrid lead iodide. $[18,19]$ Such emission offers rich opportunities for discovering single phase white light emitters such as $\mathrm{Cs}_{2} \mathrm{AgInCl}_{6}$

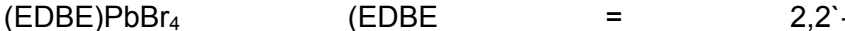
(ethylenedioxy)bis(ethylammonium)), $\quad \mathrm{C}_{4} \mathrm{~N}_{2} \mathrm{H}_{14} \mathrm{PbBr}_{4}$ (AMP) $\mathrm{PbBr}_{5} .1 / 2 \mathrm{H}_{2} \mathrm{O}$ (AMP $=1$-(2-aminoethyl)piperazine) or (TDMP)PbBr 4 (TDMP=trans-2,5-dimethylpiperaziniium). ${ }^{[16,17,20-24]}$ The Color Rendering Index (CRI) of these materials can reach high values $(\sim 70-90)$ owing to the broadband emission. ${ }^{[16,23,25]}$ However, in some color-critical high-level applications, such as jewelry, photography, museum and surgery, an ultra-high CRI is required. ${ }^{[26]}$ Through the comparison of the resulting emissions with solar spectrum (Figure S1), one can observe that such emitters are not satisfactory for these applications, because of the lack of either blue or/and red components. Thus, adding an appropriate emission band through doping can be an interesting strategy to improve the color rendering of such light source.

Doping activators such as $\mathrm{Mn}^{2+}, \mathrm{Tb}^{3+}, \mathrm{Eu}^{2+}$ or $\mathrm{Ce}^{3+}$ have been reported to generate broadband photoluminescence in hybrid lead halides. ${ }^{[14,27-32]}$ Of particular interest, $\mathrm{Mn}^{2+}$ can be doped in most of the hybrid lead halides by substituting the metal element. ${ }^{[14,31,33]}$ This ion is commonly used to improve the photoluminescence quantum yield (PLQY) or/and the stability of perovskite lattices. ${ }^{[14,34,35]}$ In addition, an improvement of the CRI is usually observed. ${ }^{[14,36]}$ These ions doped in hybrid meta halides always have an emission in the red region owing to the ${ }^{4} T_{1}$ to ${ }^{4} A_{1}$ transition. ${ }^{[14,34-37]}$ Hence, materials with emission bands covering the blue and green spectral region and lacking a red emission could be optimized through $\mathrm{Mn}$ doping. In this context, we investigate the Mn doping of (TDMP) $\mathrm{PbBr}_{4}$, which is a post-perovskite type compound we previously reported. ${ }^{[17]}$ This material exhibits a broad emission at $510 \mathrm{~nm}$, and we demonstrate that such $\mathrm{Mn}$ doping can lead to an ultra-high color rendering and high efficiency white light.

\section{Results and Discussion}

All the samples were synthesized in solution, and numbered TPbBr- $x(x=0,1,2,3,4,5)$ for increasing amount of $M n$ based reactant (Elemental ratio $\mathrm{Mn} / \mathrm{Pb}=0 / 100,4 / 96,6 / 94,8 / 92,10 / 90$, $12 / 88$, respectively) (Table $\mathrm{S} 1$ ). Interestingly, the hypothetical $\mathrm{Mn}$ based compound (TDMP) $\mathrm{MnBr}_{4}$ could not be synthesized in the same experimental conditions (Figure S2). This absence of crystallization is probably due to large differences between $\mathrm{Mn}^{2+}$ and $\mathrm{Pb}^{2+}$ radii. ${ }^{[38]}$ The structure of (TDMP) $\mathrm{PbBr}_{4}$ is shown in Figure 1a. This one-dimensional hybrid metal halide material is built of post-perovskite-type chains, which are formed by the $\mathrm{PbBr}_{6}{ }^{4-}$ octahedra connected with each other via edges and corners. ${ }^{[17]}$ These chains are not ordered at long range. The powder X-ray diffraction (XRD) patterns, which are all identical to the simulated pattern, confirm the purity of the as prepared samples (Figure 1c). In addition, no shift of XRD peaks can be observed. Generally, due to the substitution of $\mathrm{Pb}^{2+}$ (ionic radii, $1.19 \AA$ ) by smaller $\mathrm{Mn}^{2+}$ (ionic radii, $0.67 \AA$ ), XRD peaks can shift 
to higher angles. ${ }^{[39]}$ However, for low concentrations of $\mathrm{Mn}$ dopants, the influence of $\mathrm{Mn}$ on the structure is negligible and cannot be observed by X-ray diffraction. ${ }^{[40]}$ To confirm the absence of amorphous phases, solid-state NMR was carried out. As one can see on Figure S3, no differences can be observed for the samples without or with Mn doping showing that such doping does not influence the environments of lead and organic molecules. The typical TEM images for (TDMP) $\mathrm{PbBr}_{4}$ and (TDMP) $\mathrm{PbBr}_{4}: \mathrm{Mn}$ show similar needle-like particles confirming that the photoluminescence properties are not influenced by the microstructure (Figure 1b). To quantify the concentration of $\mathrm{Mn}$, inductively coupled plasma optical emission spectroscopy (ICP-OES) was performed (Table S2). Surprisingly, the results showed that the limit of solubility of $\mathrm{Mn}$ into the lattice is very low (ca. $0.030 \%$ ). Higher concentrations of $\mathrm{Mn}$ in (TDMP) $\mathrm{PbBr}_{4}$ host cannot be obtained by increasing the ratio $\mathrm{MnO}: \mathrm{PbBr}_{2}$.

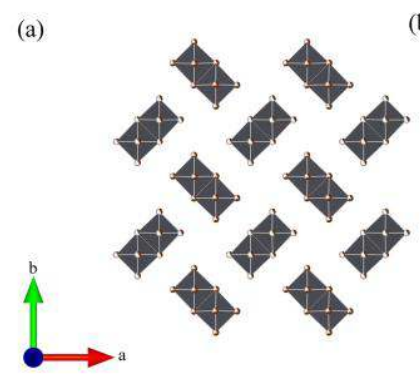

(b)
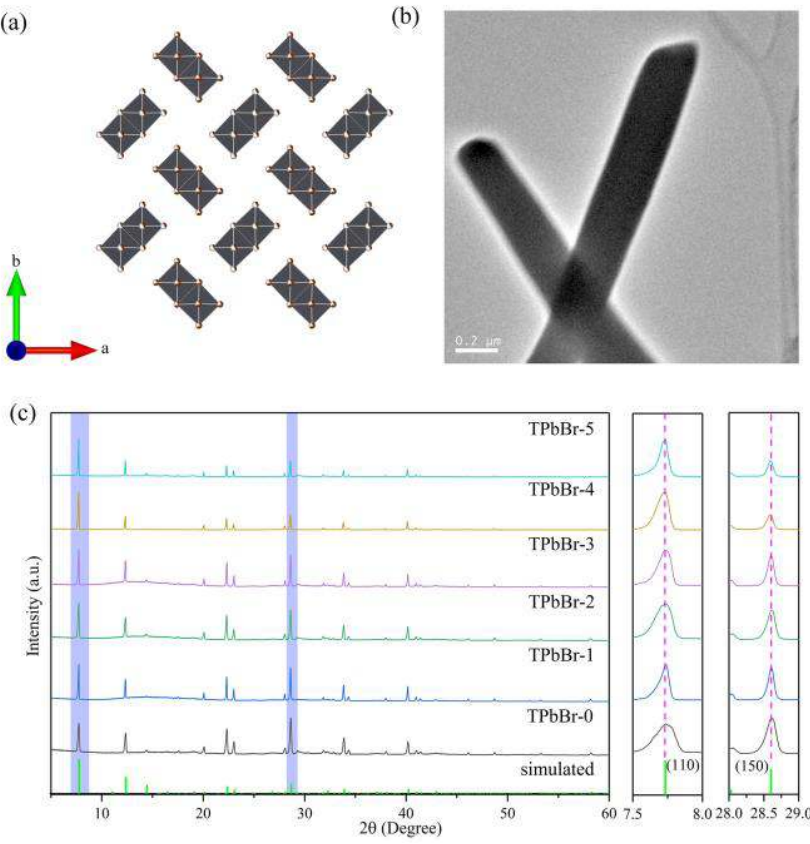

Figure 1. (a) Crystal structure of (TDMP) $\mathrm{PbBr}_{4}$, (b)TEM image for (TDMP)PbBr4, (c) Powder X-ray diffraction patterns for the different (TDMP)PbBr $4:$ Mn samples.

UV-visible diffuse reflectance spectra of (TDMP) $\mathrm{PbBr}_{4}: \mathrm{Mn}$ were collected and converted into absorption spectra using the Kubelka-Munk function (Figure 2a). The strongest absorption at $360 \mathrm{~nm}$ is attributed to the free excitons with vibronic components. No significant changes (except small changes in the relative intensities of the vibronic components) is observed upon doping suggesting an absorption originating almost exclusively from the host. The photoluminescence excitation $(\mathrm{PLE})$ and emission (PL) spectra of the $\mathrm{Mn}^{2+}$-doped (TDMP) $\mathrm{PbBr}_{4}$ phosphors with different Mn contents, are shown in Figures $2 \mathrm{~b}$. For (TDMP) $\mathrm{PbBr}_{4}$, the photoluminescence spectra show a broadband emission from $400 \mathrm{~nm}$ to $750 \mathrm{~nm}$, which originates from the self-trapped excitons (STE) states. In Mndoped samples, an additional broadband at $640 \mathrm{~nm}$ from the ligand field transition of $\mathrm{Mn}^{2+}\left({ }^{4} \mathrm{~T}_{1}\right.$ to $\left.{ }^{6} \mathrm{~A}_{1}\right)$ appears, and its intensity increases with the content of $\mathrm{Mn}$ up to $0.030 \%$ (the limit of $\mathrm{Mn}$ solubility in (TDMP) $\mathrm{PbBr}_{4}$ ). The excitation spectra of (TDMP) $\mathrm{PbBr}_{4}$ and (TDMP) $\mathrm{PbBr}_{4}$ : Mn report no significant change. All the samples have a very broad excitation range from $250 \mathrm{~nm}$ to $400 \mathrm{~nm}$, which indicates that all the samples can be effectively excited by near-UV light. Interestingly, the excitation spectra of (TDMP)PbBr 4 : Mn at $\lambda_{\mathrm{em}}=510 \mathrm{~nm}$ and $\lambda_{\mathrm{em}}=640 \mathrm{~nm}$ are very similar suggesting an identical origin of the self-trapped emission and $\mathrm{Mn}^{2+}$ emission (Figure 2b). Concerning the photoluminescence properties of the materials, the color rendering index of (TDMP) $\mathrm{PbBr}_{4}$ reaches 77.[17] This value is relatively high, which can be used in daily lighting, but the lack of emission in the red region makes this phosphor limited for applications in which ultra-high color rendering is required (Figure 2d). However, upon Mn doping, the emission at $640 \mathrm{~nm}$ increases and the CRI of the (TDMP) $\mathrm{PbBr}_{4}: \mathrm{Mn}$ is greatly enhanced (Table S3). In addition, the corresponding correlated color temperatures (CCT) can be tuned from $7034 \mathrm{~K}$ to $4188 \mathrm{~K}$ (Figure 2c), and the photoluminescence quantum yield (PLQY) can reach $68 \%$ (Table S3). Among these samples, TPbBr-3 (Mn content of $0.027 \%$ ) is particularly interesting. Its CRI reaches 96 corresponding to the record $\mathrm{CRI}$ for a single phase emitter ${ }^{[25,41,42]}$ the $\mathrm{CIE}$ coordinate $(0.330,0.365)$ corresponds to pure white light, and the PLQY reaches $60 \%$ which is a new record for lead halide white emitter (Figure 2e). ${ }^{[17,20-22,25,43]}$ Even if other compounds such as $\left(\mathrm{H}_{2} \mathrm{DABCO}\right)\left(\mathrm{Pb}_{2} \mathrm{Cl}_{6}\right)$ and $\left(\mathrm{C}_{5} \mathrm{H}_{7} \mathrm{~N}_{2}\right)_{2} \mathrm{SnBr}_{4}$ were reported with $\mathrm{CRI}$ close to 96 , the intensity of their emissions remained very low $(P L Q Y<3 \%)^{[41]}$ or did not correspond to a pure white light. ${ }^{[42]}$ Besides, it is important to note that the CRI value is merely an index which only considers the first eight (R1-R8) color samples. For $\mathrm{TPbBr}-3$, all the values from R1 to R14 are above 90, which is exceptional for a single phase phosphor (Figure 2(d)). For example, R9 reflecting the color reproduction in the strong red region, is very important for biomedical and painting applications ${ }^{[12]}$. In commercial blue-chip LEDs, R9 is typically very low (i.e. less than 20 ), while this value reaches 92 for (TDMP) $\mathrm{PbBr}_{4}$ : Mn. ${ }^{[4]}$ Thus, the phosphors (TDMP)PbBr $4:$ Mn demonstrate an excellent white-light emission with a high PLQY, an ultra-high CRI value and a tunable CCT value which are important parameters for use as phosphor in wLEDs. Figure S4 shows the evolution of the photoemission from (TDMP)PbBr $4:$ Mn with temperature (up to $150{ }^{\circ} \mathrm{C}$ ). When the temperature increases, the PL intensity decreases without significant modification of emission shape. This evolution is similar to the ones previously described for other hybrid lead halides. ${ }^{[14,45]}$ The thermal degradation of (TDMP)PbBr 4 : Mn was analyzed by differential scanning calorimetry and thermogravimetry. In addition, the moisture stability under low/high temperatures was investigated by carrying out XRD and $\mathrm{PL}$ measurements before and after treatments in air at low/high temperatures for different durations (Figure S5 and Figure S6). Both undoped and doped materials show good thermal stability (the thermal decomposition occurs above 300 ${ }^{\circ} \mathrm{C}$ ) and good moisture stability (no change in the purity of samples or PL properties after treatments under air at room temperature for three months or at $100^{\circ} \mathrm{C}$ for one week). To highlight the potential of such phosphor in solid-state lighting, a white LED was fabricated. $\mathrm{TPbBr}-3$ was mixed with resin and coated on UV LED chips ( $365 \mathrm{~nm}$ ) and could exhibit a bright white light (Figure $3 \mathrm{c}$ and Figure S7). 

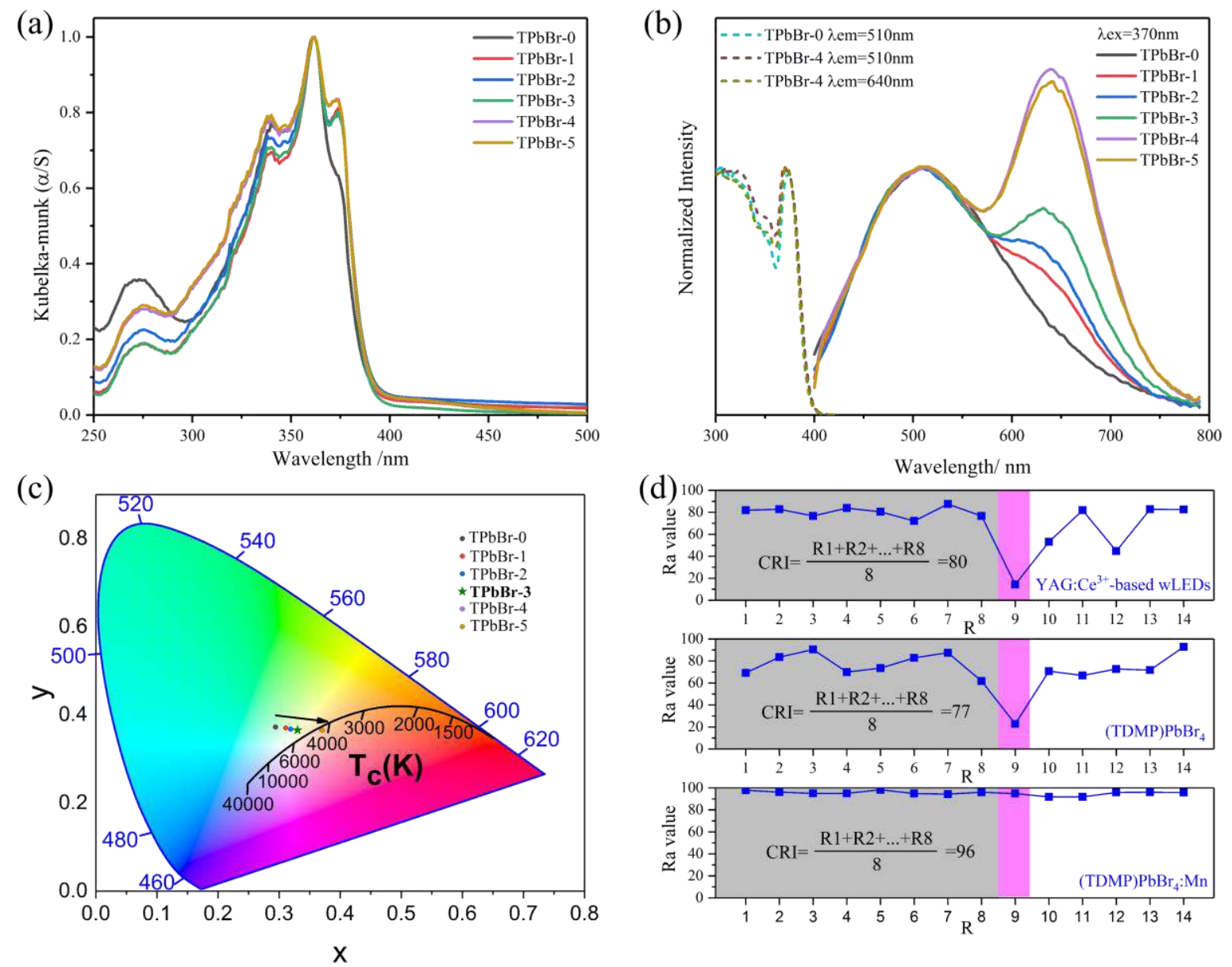

Figure 2. Optical properties of (TDMP) $\mathrm{PbBr}_{4}$ and (TDMP) $\mathrm{PbBr}_{4}$ : Mn phosphors: (a) Kubelka-Munk absorption spectra, (b) photoluminescence excitation ( $\mathrm{PLE}$ ) and photoluminescence emission (PL) spectra, (c) CIE coordinates and (d) comparison of CRI values from R1 to R14 for commercial YAG: Ce ${ }^{3+}$-based wLEDs ${ }^{[12]}$, (TDMP) $\mathrm{PbBr}_{4}(\mathrm{TPbBr}-0)$, and (TDMP)PbBr $4: 0.027 \% \mathrm{Mn}(\mathrm{TPbBr}-3)$.

To investigate the origin of the intense red emission from such low amount of $\mathrm{Mn}$ dopant, the tin analogues (TDMP) $\mathrm{SnBr}_{4}$ and (TDMP)SnBr $4: \mathrm{Mn}^{2+}$ were synthesized and characterized (Figure S8). (TDMP) $\mathrm{SnBr}_{4}$ crystallizes in the same structure as ( $\mathrm{TDMP} \mathrm{PbBr}_{4}$, but its photoemission $(430 \mathrm{~nm})$ is very weak (Figure S9). Upon doping $\mathrm{Mn}$ into (TDMP) $\mathrm{SnBr}_{4}$, the emission originating from the ligand field transition of $\mathrm{Mn}^{2+}\left({ }^{4} \mathrm{~T}_{1}\right.$ to $\left.{ }^{6} \mathrm{~A}_{1}\right)$ appears but remains weak (intensity comparable to the emission from the host). Such results further confirm that the emission of $\mathrm{Mn}$ in hybrid metal halides is strongly coupled with the excitons. In order to exhibit an intense red emission from the Mn dopants, the photoluminescence of the host (i.e. excitonic emission) should also be intense. Interestingly, very low amount of dopants (less than $0.030 \%$ ) are sufficient to obtain high PLQYs. Such enhanced exciton-dopant exchange coupling interactions is probably due to high quantum confinement as excitons can diffuse in only one dimension. ${ }^{[33]}$

In the literature, the exact nature of exciton-dopant coupling for hybrid metal halides remains unclear. Some authors reported that energy transfer is occurring only between free excitons and $\mathrm{Mn}$ because the self-trapped exciton states are indirect transient states with unpopulated ground states that could block electron exchange in Dexter energy transfer. ${ }^{[14,15]}$ At the opposite, Zhou et al. reported that the Mn emission originates from both STE and FE states because both related emissions are affected by the $\mathrm{Mn}$ doping. ${ }^{[37]}$ Thus, to unravel the mechanism of luminescence in our $\mathrm{Mn}$ doped lead halide, temperature dependent $\mathrm{PL}$ measurements were performed for (TDMP) $\mathrm{PbBr}_{4}$ (Figure S10) and (TDMP)PbBr ${ }_{4}:$ Mn (Figure 3(a-b)). ${ }^{[3]}$ At low temperature (below ca. 120K), only one emission band originating from self-trapped excitons can be observed for both doped and undoped materials. At higher temperatures (above ca. 120K), the STE emission decreases and a new emission band attributed to the ligand field transition $\left({ }^{4} \mathrm{~T}_{1}\right.$ to $\left.{ }^{6} \mathrm{~A}_{1}\right)$ of $\mathrm{Mn}^{2+}$ appears for the doped phosphor (Figure 3(a)). Thus, a mechanism describing the equilibrium between FE, STE and Mn emissions can be proposed (Figure $3(\mathrm{~d}-\mathrm{e})$ )). At low temperature and under UV light, free excitons are photogenerated and get trapped in the STE states (the trapping of excitons is thermally activated at temperatures as low as $77 \mathrm{~K}) \cdot{ }^{[17,46]}$ However, no luminescence from $\mathrm{Mn}$ is observed because the associated ligand field transition is dipole forbidden. As the temperature increases, the detrapping of excitons is thermally activated, 
(a)

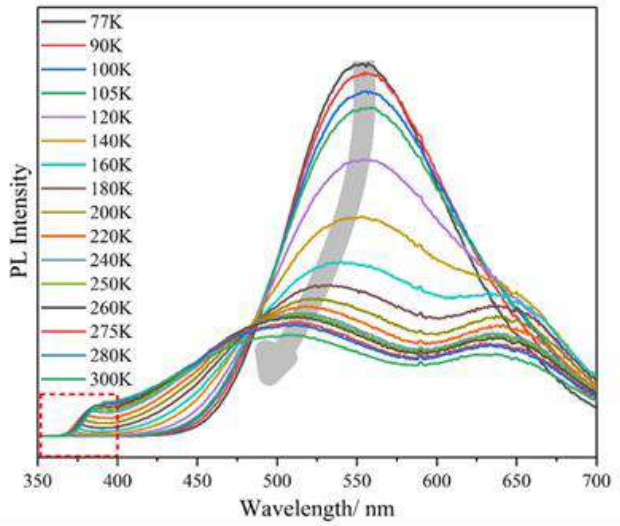

(d)
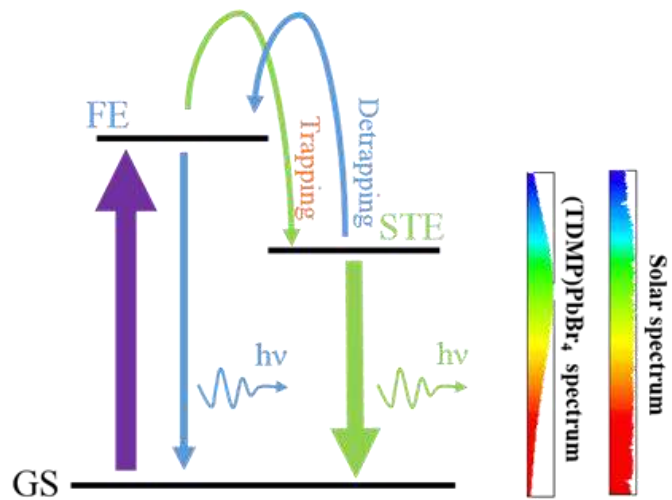

(b)

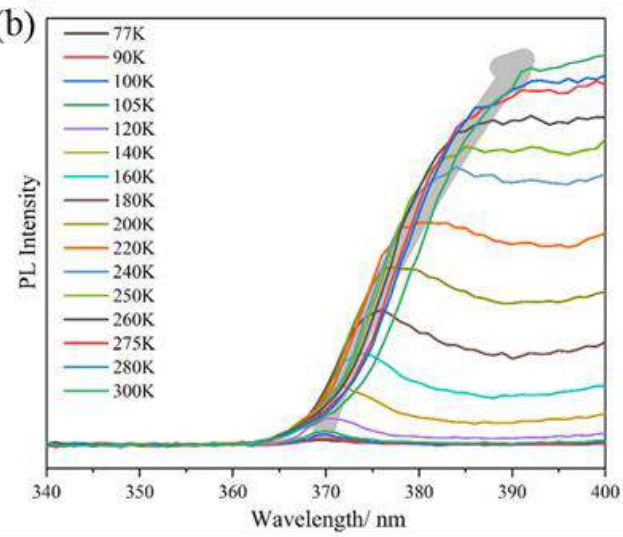

(c)
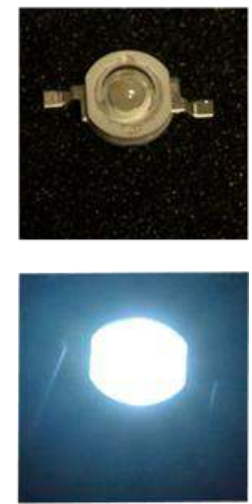

(e)

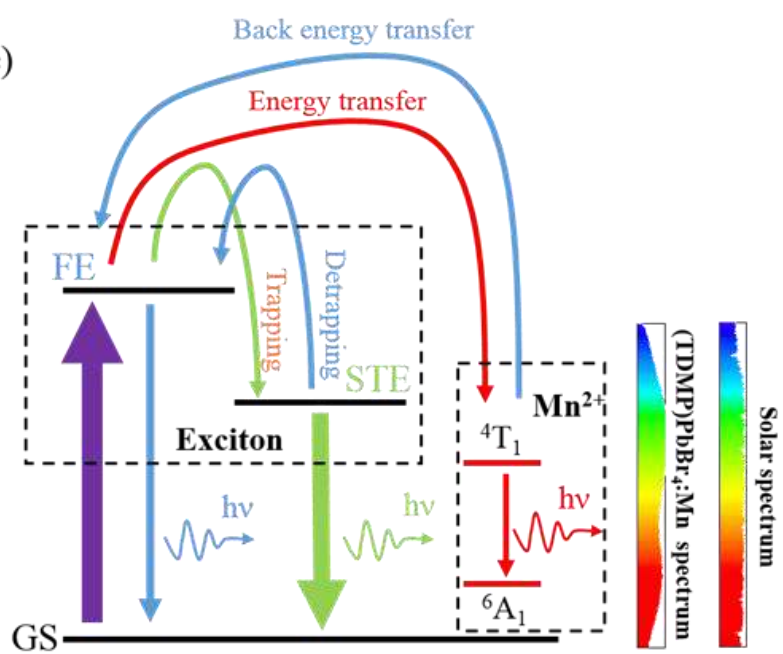

Figure 3. (TPbBr-3). Photoluminescence properties of TPbBr-3 (a) Evolution of STE emission with temperature, (b)Evolution of FE emission with temperature, (c) white LED fabricated from a n-UV LED chip $(365 \mathrm{~nm}$ ) coated with $\mathrm{TPbBr}-3$ phosphor. Mechanism of luminescence for (d) (TDMP)PbBr 4 and (e) (TDMP)PbBr 4 : Mn.

leading the FE emission to increase and the STE emission to decrease with temperature (Figure $3(a-b)) \cdot{ }^{[17]}$ In parallel, the selection rule of the forbidden transition of the $\mathrm{Mn}$ dopant is relaxed owing to vibronic coupling. ${ }^{[33]}$ Thus, the rate of energy transfer exciton-dopant increases and leads to the increase of the Mn emission with temperature. Above ca. 120K at which Mn emission appears, the evolutions with temperature of FE or STE emissions differ when comparing (TDMP) $\mathrm{PbBr}_{4}$ and (TDMP)PbBr 4 : Mn (Figure S11). These different evolutions between undoped and doped compounds can be explained by a competition of the exciton-dopant energy transfer with the recombination of the exciton. As FE and STE are in thermal equilibrium above $100 \mathrm{~K},{ }^{[46]}$ the competition between the recombination of the $\mathrm{FE}$ and the exciton-dopant energy transfer will also affect the recombination of the STE (Figure 3(e)). Interestingly, the energy transfer promotes the photoluminescence intensity as the PLQY increases with the amount of Mn (Table S3). This phenomenon could be explained by the existence of $\mathrm{Mn}^{2+}$ which provides an efficient radiative recombination pathway for the exciton $\left({ }^{4} T_{1}\right.$ to $\left.{ }^{6} A_{1}\right) \cdot{ }^{[47]}$ Thus, with an increasing concentration of $\mathrm{Mn}^{2+}$, the number of radiative recombinations of the excitons through this transition metal ion would increase resulting in the enhancement of the PLQY. Besides, as previously reported for II-VI semiconductors, the $\mathrm{Mn}^{2+}$ in hybrid lead halide would behave as a long-lived energy reservoir of excitons through back energy transfer from excited $\mathrm{Mn}^{2+}$ to the creation of excitons. ${ }^{[48,49]}$ This mechanism could also contribute to the enhancement of the PLQY.

\section{Conclusion}

In summary, doping very low amount of $\mathrm{Mn}^{2+}$ in (TDMP) $\mathrm{PbBr}_{4}$ enabled to increase the high white photoluminescence $Q Y$ for lead halides from $45 \%$ to $68 \%$. In addition, the color rendering reaches record values (96) for single phase white phosphors and a tunable CCT (from $7034 \mathrm{~K}$ to $4188 \mathrm{~K}$ ) could be obtained. Besides, new insights were provided on the mechanism of luminescence from $\mathrm{Mn}^{2+}$ doped hybrid lead halides. $\mathrm{Mn}^{2+}$ luminescence is strongly coupled with the excitons in free or self-trapped states because these both states are in thermal equilibrium. Thus, tuning the amount of $\mathrm{Mn}$ dopant enables to control the competition existing between the energy transfer dopant-exciton and the recombination of excitons. An appropriate balance between FE, STE, and energy transfer to $\mathrm{Mn}$ enables to reach a pure white emission. Thus, we believe this work could provide new ideas on how light sources with ultra-high color rendering could be designed in the future.

\section{Experimental Section}

(TDMP) $\mathrm{PbBr}_{4}: \mathrm{Mn}$ samples were synthesized from a mixture of $\mathrm{PbBr}_{2}$ (Alfa Aesar, 99.95\%), MnO (Alfa Aesar, 99.95\%) and trans-2,5-dimethylpiperazine (alfa Aesar, 98\%) added into $20 \mathrm{~mL}$ $\mathrm{HBr}$ (Alfa Aesar, 48\%). After heating under reflux for three hours 
with agitation, the solutions were cooled down and a white powder was recovered by filtration and washed with ethanol. The detailed amount of each reactant for different samples can be found in Table S1.

\section{Acknowledgements}

This work was supported by the National Agency for Research (ANR Young Researchers, ANR-16-CE08-0003-01, Combi-SSL project) and Region Pays de la Loire (Etoiles montantes en Pays de la Loire 2017, project "Découverte de pérovskites hybrides assistée par ordinateur"). The National Natural Science Foundation of China (51472188, 51521001, and 51872217), Natural Research Funds of Hubei Province (No. 2016CFB583), the Fundamental Research Funds for the Central Universities in China and the "111" project (B13035). HL Yuan thanks the China Scholarship Council (CSC) for fellowship support. We thank Carole La and the Laboratoire de Planétologie et Géodynamique for the ICP-OES analysis.

Keywords: Ultra-high color rendering $\bullet$ high photoluminescence quantum yield $\bullet$ single phase phosphor $\bullet$ hybrid lead halides

\section{References}

[1] E. F. Schubert, J. K. Kim, Science 2005, 308, 1274-1278.

[2] M. Zhao, H. Liao, M. S. Molokeev, Y. Zhou, Q. Zhang, Q. Liu, Z. Xia, Light: Science \& Applications 2019, 8, 38.

[3] H. Lian, Q. Huang, Y. Chen, K. Li, S. Liang, M. Shang, M. Liu, J. Lin, Inorganic chemistry 2017, 56, 11900-11910.

[4] S. Ye, F. Xiao, Y. X. Pan, Y. Y. Ma, Q. Y. Zhang, Materials Science and Engineering: R: Reports 2010, 71, 1-34.

[5] G.-H. Pan, H. Wu, S. He, L. Zhang, Z. Hao, X. Zhang, J. Zhang, Nanoscale 2018, 10, 22237-22251.

[6] J. Chen, Y. Zhao, Z. Mao, D. Wang, L. Bie, Materials Research Bulletin 2017, 90, 212-217.

[7] S. J. Park, H. K. Yang, B. K. Moon, Nano Energy 2019, 60, 87-94

[8] L. E. Downie, P. R. Keller, L. Busija, J. G. Lawrenson, C. C. Hull, Cochrane Database of Systematic Reviews 2019.

[9] K. M. Zielinska-Dabkowska, Make Lighting Healthier, Nature Publishing Group, 2018

[10] P. M. Pattison, J. Y. Tsao, G. C. Brainard, B. Bugbee, Nature 2018, $563,493$.

[11] Z. Tian, P. Tian, X. Zhou, G. Zhou, S. Mei, W. Zhang, X. Zhang, D. Li, D. Zhou, R. Guo, Nanoscale 2019, 11, 3489-3494

[12] P.-P. Dai, C. Li, X.-T. Zhang, J. Xu, X. Chen, X.-L. Wang, Y. Jia, X. Wang, Y.-C. Liu, Light: Science \& Applications 2016, 5, e16024.

[13] M. Shang, C. Li, J. Lin, Chemical Society Reviews 2014, 43, 13721386.

[14] C. Zhou, Y. Tian, O. Khabou, M. Worku, Y. Zhou, J. Hurley, H. Lin, B. Ma, ACS applied materials \& interfaces 2017, 9, 40446-40451.

[15] M. Han, Y. Tian, Z. Yuan, L. Zhu, B. Ma, Angewandte Chemie International Edition 2014, 53, 10908-10912.

[16] S. Brochard-Garnier, M. Paris, R. Génois, Q. Han, Y. Liu, F. Massuyeau, R. Gautier, Advanced Functional Materials 2019, 29, 1806728.

[17] R. Gautier, F. Massuyeau, G. Galnon, M. Paris, Advanced Materials 2019, 31, 1807383.

[18] A. Nagami, K. Okamura, T. Ishihara, Physica B: Condensed Matter 1996, 227, 346-348.

[19] T. Ishihara, in Optical Properties of Low-Dimensional Materials, World Scientific, 1995, pp. 288-339.
[20] J. Luo, X. Wang, S. Li, J. Liu, Y. Guo, G. Niu, L. Yao, Y. Fu, L. Gao, Q. Dong, Nature 2018, 563, 541.

[21] Z. Yuan, C. Zhou, Y. Tian, Y. Shu, J. Messier, J. C. Wang, L. J. Van De Burgt, K. Kountouriotis, Y. Xin, E. Holt, Nature communications 2017, 8, 14051.

[22] E. R. Dohner, A. Jaffe, L. R. Bradshaw, H. I. Karunadasa, Journal of the American Chemical Society 2014, 136, 13154-13157.

[23] L. Mao, P. Guo, M. Kepenekian, I. Hadar, C. Katan, J. Even, R. D. Schaller, C. C. Stoumpos, M. G. Kanatzidis, Journal of the American Chemical Society 2018, 140, 13078-13088.

[24] R. Roccanova, M. Houck, A. Yangui, D. Han, H. Shi, Y. Wu, D. T. Glatzhofer, D. R. Powell, S. Chen, H. Fourati, ACS Omega 2018, 3 , 18791-18802

[25] K. Thirumal, W. K. Chong, W. Xie, R. Ganguly, S. K. Muduli, M. Sherburne, M. Asta, S. Mhaisalkar, T. C. Sum, H. S. Soo, Chemistry of Materials 2017, 29, 3947-3953.

[26] C. Ji, S. Wang, L. Li, Z. Sun, M. Hong, J. Luo, Advanced Functional Materials 2019, 29, 1805038

[27] X. Zhang, L. Li, Z. Sun, J. Luo, Chemical Society Reviews 2019, 48, 517-539.

[28] J.-S. Yao, J. Ge, B.-N. Han, K.-H. Wang, H.-B. Yao, H.-L. Yu, J.-H. Li, B.-S. Zhu, J.-Z. Song, C. Chen, Journal of the American Chemical Society 2018, 140, 3626-3634

[29] Q. Hu, Z. Li, Z. Tan, H. Song, C. Ge, G. Niu, J. Han, J. Tang, Advanced Optical Materials 2018, 6, 1700864

[30] R. Yuan, L. Shen, C. Shen, J. Liu, L. Zhou, W. Xiang, X. Liang, Chemical communications 2018, 54, 3395-3398.

[31] D. Parobek, B. J. Roman, Y. Dong, H. Jin, E. Lee, M. Sheldon, D. H. Son, Nano letters 2016, 16, 7376-7380.

[32] A. Biswas, R. Bakthavatsalam, J. Kundu, Chemistry of Materials 2017 29, 7816-7825.

[33] R. Bakthavatsalam, A. Biswas, M. Chakali, P. R. Bangal, B. P. Kore, J. Kundu, The Journal of Physical Chemistry C 2019, 123, 4739-4748.

[34] S. Zou, Y. Liu, J. Li, C. Liu, R. Feng, F. Jiang, Y. Li, J. Song, H. Zeng, M. Hong, Journal of the American Chemical Society 2017, 139 , 11443-11450.

[35] F. Locardi, M. Cirignano, D. Baranov, Z. Dang, M. Prato, F. Drago, M Ferretti, V. Pinchetti, M. Fanciulli, S. Brovelli, Journal of the American Chemical Society 2018, 140, 12989-12995.

[36] G. Pan, X. Bai, W. Xu, X. Chen, D. Zhou, J. Zhu, H. Shao, Y. Zhai, B. Dong, L. Xu, ACS applied materials \& interfaces 2018, 10, 3904039048.

[37] G. Zhou, X. Jiang, M. Molokeev, Z. Lin, J. Zhao, J. Wang, Z. Xia, Chemistry of Materials 2019.

[38] R. D. Shannon, Acta crystallographica section A: crystal physics, diffraction, theoretical and general crystallography 1976, 32, 751-767. A. Yangui, R. Roccanova, Y. Wu, M.-H. Du, B. Saparov, The Journal of Physical Chemistry C 2019, 123, 22470-22477.

[40] T. Sheikh, A. Nag, The Journal of Physical Chemistry C 2019, 123, 9420-9427.

[41] G.-E. Wang, G. Xu, M.-S. Wang, L.-Z. Cai, W.-H. Li, G.-C. Guo, Chemical science 2015, 6, 7222-7226.

[42] A. Yangui, R. Roccanova, T. M. McWhorter, Y. Wu, M.-H. Du, B. Saparov, Chemistry of Materials 2019, 31, 2983-2991.

[43] A. Yangui, D. Garrot, J.-S. Lauret, A. Lusson, G. Bouchez, E. Deleporte, S. Pillet, E.-E. Bendeif, M. Castro, S. Triki, The Journal of Physical Chemistry C 2015, 119, 23638-23647.

[44] Y. Narukawa, J. Narita, T. Sakamoto, T. Yamada, H. Narimatsu, M. Sano, T. Mukai, physica status solidi (a) 2007, 204, 2087-2093.

[45] B. T. Diroll, G. Nedelcu, M. V. Kovalenko, R. D. Schaller, Advanced Functional Materials 2017, 27, 1606750.

[46] R. Gautier, M. Paris, F. Massuyeau, Journal of the American Chemical Society 2019, 141, 12619-12623

[47] Y. Peng, L. Li, C. Ji, Z. Wu, S. Wang, X. Liu, Y. Yao, J. Luo, Journal of the American Chemical Society 2019, 141, 12197-12201.

[48] H.-Y. Chen, D. H. Son, Israel Journal of Chemistry 2012, 52, 10161026.

[49] R. Beaulac, P. I. Archer, J. van Rijssel, A. Meijerink, D. R. Gamelin, Nano letters 2008, 8, 2949-2953. 